\title{
Focused Arrays Beamforming
}

\author{
Oleksandr Mazurenko and Yevhenii Yakornov \\ Institute of Telecommunication Systems, \\ National Technical University of Ukraine "Kyiv Polytechnic Institute" \\ Ukraine
}

\section{Introduction}

Over the past two decades many articles devoted to the antenna systems focused in it's near-field zone (NFZ) or intermediate-field zone (IFZ) and their application in medical engineering, geology, materials and environment sensing, RFID, energy transfer technologies were published. The development of this theory allows to improve the quality level of technique and to expand applicability of the focused antenna systems, for example, in the telecommunications engineering.

Development of the focused antennas theory began in the late 1950's. The first collection of papers that describe the properties of the focused antenna, edited by Hansen, was printed in 1964 (Hansen, 1964). Further development of this theory was not so active until the 1990's. Recent works in this area relates only to the practical application and realization of the focusing effect (Herben, 1999; Hristov, 2004; Karimkashi \& Kishik, 2008; Rudolph \& Grbic, 2008; etc.) and finding of the methods of improving the focused antennas performance (Hussain, 2004, 2008; Karimkashi \& Kishik, 2009; etc.).

Reference materials for this paper are based on a current technical level, accordingly to URSI and IEEE papers, within the limits of knowledge of the near-field and the intermediate field diffraction theory, focal areas forming on the plane apertures radiation axis and signal processing methods of the focused arrays for various environments scanning.

The authors decided that the reference materials are insufficiently exploring the problem for wider and more flexible usage of the three-dimensionally directional signal transmission phenomenon due to an incompleteness of the focused antenna arrays (FAA) theory. The given incompleteness is revealing as a high level of calculations for obtaining the exact aperture phase distribution, inaccuracy and deficiency of theoretical models, that does not allow to use qualitatively the focused energy transmission to a certain area of space at a wide range of angles in azimuth and elevation planes.

The basis of this chapter is the results of research led for the purpose of improving FAA theory for its further usage in the telecommunication engineering that cannot be done without increasing of FAA performance. The research materials are devoted to a wide range of FAA structures with different types of radiator and to the methods of FAA directivity improving with a purpose to increase the 3-dimensional gain performance of antenna arrays at a wide range of angles in azimuth and elevation planes.

This chapter is organized as follows. Section 2 is devoted to a new approach that better reveals the principles of FAA radiation pattern forming, including FAA beamforming with 
various radiators types and allocation. FAA directivity improving methods are considered in Section 3. FAA possible applications for a short distance wireless communication are described in Section 4. Concluding remarks and future activities are collected in Section 5.

\section{Focused Antenna Arrays radiation patterns}

When writing this section the authors did not attempt to create a new huge mathematical model that would describe the distribution of field or power radiated by different types of antennas, but instead of it to find new approaches for better describing the characteristics of focused antennas. If the reader wants to see the detailed, but approximated by Fresnel description of a field radiated by focused aperture or its focusing properties, he can refer to an existing theory (Chu, 1971; Fenn, 2007; Graham, 1983; Hansen, 1964, 1985, 2009; Laybros et al., 2005; Malyuskin \& Fusco, 2009; Narasimhan \& Philips, 1987a, 1987b; Polk, 1956).

Generally consider the antenna arrays of linear structure as that is sufficient to study properties of FAA. Thus all tasks of study of FAA radiation pattern synthesis are sufficient to be done in its azimuth plane, while considering the linear antenna array.

\subsection{Geometric models}

In this subsection we present geometric models of different structures of arrays. In the next subsections we will describe radiation patterns of arrays based on this models.

For a start, consider the problem of finding an expression for a linear array with equivalent spaced radiators (LAESR) without any mathematical approximation (Fraunhofer or Fresnel), where phase shifts between array elements and the array phase center are determined by two exact components: the phase shift by angle and the phase shift by distance. The geometry model of LAESR is shown in Fig.1, where $d$ - array element spacing between $2 N+1$ radiators with number $n$, normal vector to the array is polar axis or the starting point of polar coordinates in which the problem is solved. An important factor is the location of the phase center, which contains the polar axis. Let phase center be located in the LAESR center element with $n=0$. Then location of observation point is described by azimuth $\theta$ and distance $R$ relatively to the array phase center. Thereby all equations related to the right side elements (RSE) with $n_{R S E}=1 \ldots N=n$ and the left side elements (LSE) with $n_{L S E}=-1 \ldots-N=-n$ from the phase center differ by indexes and content. Then $\Delta\left(n_{L S E}\right), \Delta\left(n_{R S E}\right)$ are spatial shifts between phase center and LSE, RSE respectively; $\mathrm{v}\left(n_{L S E}\right), \mathrm{v}\left(n_{R S E}\right)$ are angles between phase center and LSE, RSE with number $n$ respectively in observation point; $\theta\left(n_{L S E}\right), \theta\left(n_{R S E}\right)$ are azimuths of observation point from LSE, RSE respectively.

Obtain the two equation systems using elementary trigonometry for LAESR (fig.1):

$$
\begin{gathered}
\left\{\begin{array}{l}
\frac{d n_{L S E}}{\sin \left(v\left(n_{L S E}\right)\right)}=\frac{R+\Delta\left(n_{L S E}\right)}{\cos (\theta)}=\frac{R}{\cos \left(\theta+v\left(n_{L S E}\right)\right)} \\
\frac{d n_{R S E}}{\sin \left(v\left(n_{R S E}\right)\right)}=\frac{R+\Delta\left(n_{R S E}\right)}{\cos (\theta)}=\frac{R}{\cos \left(\theta-v\left(n_{R S E}\right)\right)}
\end{array}\right. \\
\left\{\begin{array}{l}
\Delta\left(n_{L S E}\right)=\sqrt{R^{2}+\left(d n_{L S E}\right)^{2}+2 R d n_{L S E} \sin (\theta)}-R \\
\Delta\left(n_{R S E}\right)=\sqrt{R^{2}+\left(d n_{R S E}\right)^{2}-2 R d n_{R S E} \sin (\theta)}-R
\end{array}\right.
\end{gathered}
$$


From equations (1), (2), difference between the expressions for the RSE and LSE is due to using the number sign of array element.

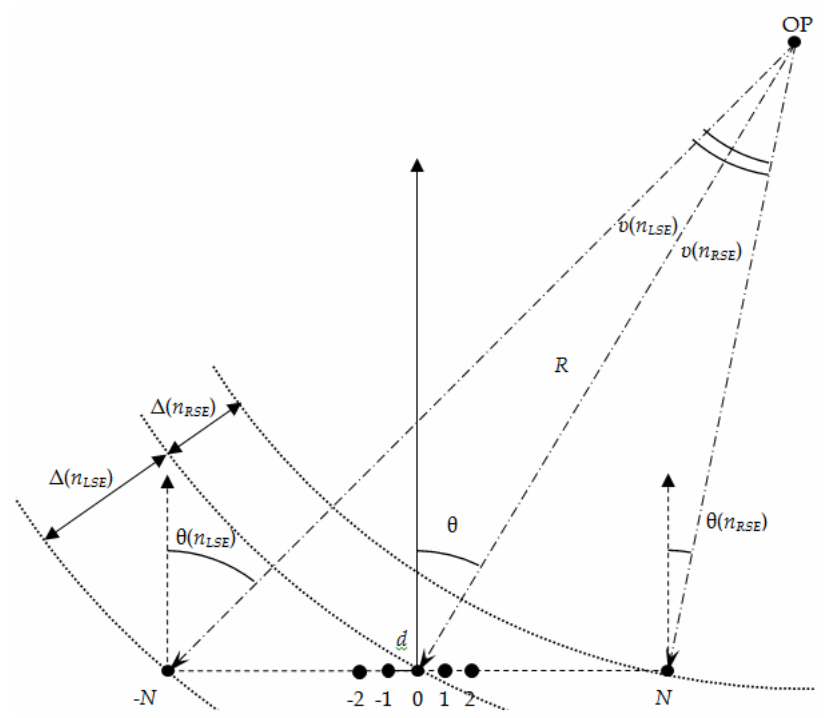

Fig. 1. The geometric model of LAESR

Expression (2) is regular for the spatial shift $\Delta$ (Fenn, 2007; Hansen, 1964), then using (1) to solve the problem mentioned before.

Obtain the equation systems for $\Delta\left(n_{L S E}\right), \Delta\left(n_{R S E}\right)$ and $\mathrm{v}\left(n_{L S E}\right), \mathrm{v}\left(n_{R S E}\right)$ from (1):

$$
\begin{gathered}
\left\{\begin{array}{l}
\Delta\left(n_{L S E}\right)=\frac{d n_{L S E} \cos (\theta)}{\sin \left(v\left(n_{L S E}\right)\right)}-R \\
\Delta\left(n_{R S E}\right)=\frac{d n_{R S E} \cos (\theta)}{\sin \left(v\left(n_{R S E}\right)\right)}-R
\end{array}\right. \\
\left\{\begin{array}{l}
\Delta\left(n_{L S E}\right)=d n_{L S E}\left(\sin (\theta)+\cos (\theta) \tan \left(\frac{v\left(n_{L S E}\right)}{2}\right)\right) \\
\Delta\left(n_{R S E}\right)=d n_{R S E}\left(-\sin (\theta)+\cos (\theta) \tan \left(\frac{v\left(n_{R S E}\right)}{2}\right)\right)
\end{array}\right. \\
v\left(n_{L S E}\right)=\operatorname{arccot}\left(\frac{R}{d n_{L S E} \cos (\theta)}+\tan (\theta)\right), v\left(n_{L S E}\right) \in\left[0 ; \frac{\pi}{2}\right] \\
v\left(n_{R S E}\right)=\operatorname{arccot}\left(\frac{R}{d n_{R S E} \cos (\theta)}-\tan (\theta)\right), v\left(n_{R S E}\right) \in\left[0 ; \frac{\pi}{2}\right]
\end{gathered} .
$$

Then equation (4) can be written as: 


$$
\left\{\begin{array}{l}
\Delta\left(n_{L S E}\right)=d n_{L S E}\left(\sin (\theta)+\cos (\theta) \tan \left(0.5 \operatorname{arccot}\left(\frac{R}{d n_{L S E} \cos (\theta)}+\tan (\theta)\right)\right)\right) \\
\Delta\left(n_{R S E}\right)=d n_{R S E}\left(-\sin (\theta)+\cos (\theta) \tan \left(0.5 \operatorname{arccot}\left(\frac{R}{d n_{R S E} \cos (\theta)}-\tan (\theta)\right)\right)\right)
\end{array} .\right.
$$

Another important parameter for radiation pattern calculating is the observation point azimuth $\theta(n)$. The expressions for $\theta\left(n_{L S E}\right), \theta\left(n_{R S E}\right)$ are:

$$
\left\{\begin{array}{l}
\theta\left(n_{L S E}\right)=\arcsin \left[\frac{R \sin (\theta)+d n_{L S E}}{\sqrt{R^{2}+\left(d n_{L S E}\right)^{2}+2 R d n_{L S E} \sin (\theta)}}\right] \\
\theta\left(n_{R S E}\right)=\arcsin \left[\frac{R \sin (\theta)-d n_{R S E}}{\sqrt{R^{2}+\left(d n_{R S E}\right)^{2}-2 R d n_{R S E} \sin (\theta)}}\right]
\end{array} .\right.
$$

From expressions (5), (6), (7) for left side and right side antenna elements, the phase center location affects the spatial shifts distribution in NFZ and IFZ can be concluded. General equation for $\Delta(n), \mathrm{v}(n)$ and $\theta(n)$, where $n \in[-N ; N], n \in \mathbf{Z}$, using (5), (6), (7) can be written as:

$$
\begin{gathered}
\Delta(n)=d n\left(\cos (\theta) \tan \left(0.5 \operatorname{arccot}\left(\frac{R}{d n \cos (\theta)}-\tan (\theta)\right)\right)-\sin (\theta)\right) ; \\
v(n)=\operatorname{arccot}\left(\frac{R}{d n \cos (\theta)}-\tan (\theta)\right), v(n) \in\left[0 ; \frac{\pi}{2}\right] ; \\
\theta(n)=\arcsin \left[\frac{R \sin (\theta)-d n}{\sqrt{R^{2}+(d n)^{2}-2 R d n \sin (\theta)}}\right] .
\end{gathered}
$$

Expressions (8), (9), (10) are necessary in calculating LAESR radiation pattern.

Equation (8) of spatial shift obtained in the form of expression $\Delta=\Delta_{R}+\Delta_{\theta}$ is useful for FAA with separate phase steering by distance and by angular coordinates synthesis, it also helps to reduce the level of computational operations to calculate required phase distribution.

In elevation plane of spherical coordinates spatial shifts of LAESR mentioned before are equal at different elevation angles.

The linear structure is most often used in the construction of antenna arrays. However, there are other structures that as the best create the Rayleigh or the Fresnel diffraction field. There are polygonal structures where all elements are radiating inside the polygon. It should be noted that antenna arrays of polygonal structures have their own natural focus.

Next step is finding an expression for spatial shifts between phase center and elements of polygonal antenna array (PAA) using vector analysis theory. Place phase center of PAA in its natural focus to solve this task since the problem of obtaining equations between the PAA parameters is complex. 
The geometric model of PAA is shown in Fig.2, where 1...n-th antenna location points, $d_{i j}-$ distance between $i$-th and $j$-th antenna element of PAA, 0 - centre of the polygon and phase center of PAA, $0^{\prime}$ - observation point, $\mathbf{L}$ - polar axis of PAA in polar coordinate, where the problem is being solved; $\mathbf{R}_{n}\left(\left|\mathbf{R}_{n}\right| ; \theta_{R n}\right)$ - vector of $n$-th antenna signal in phase center and its polar axis, where $\left|\mathbf{R}_{n}\right|$ - spatial shift between $n$-th antenna and phase center; $\Delta\left(|\Delta| ; \theta_{\Delta}\right)$ vector from phase center to observation point, where $|\Delta|$ - spatial shift between phase center and observation point, $\theta_{n}$ - azimuth of observation point in $n$-th antenna. Then the spatial shift between $n$-th antenna and observation point:

$$
\Delta_{n}=\sqrt{\left|\mathbf{R}_{n}\right|^{2}+|\boldsymbol{\Delta}|^{2}+\left|\mathbf{R}_{n}\right||\boldsymbol{\Delta}| \cos \left(\theta_{R n}-\theta_{\Delta}\right)}-\left|\mathbf{R}_{n}\right| .
$$

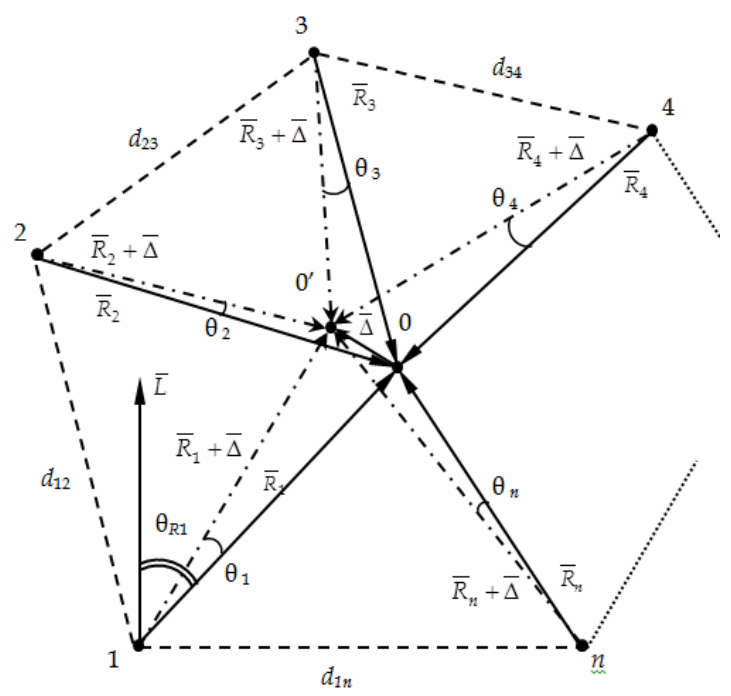

Fig. 2. Geometry model of PAA

Equations (11) can be written in form of $\Delta=\Delta_{R}+\Delta_{\theta}$, as follows:

$$
\Delta_{n}=|\Delta|\left(\sin \left(\theta_{R n}-\theta_{\Delta}\right) \tan \left(0.5 \operatorname{arccot}\left(\frac{\left|\mathbf{R}_{n}\right|}{|\Delta| \sin \left(\theta_{R n}-\theta_{\Delta}\right)}+\cot \left(\theta_{R n}-\theta_{\Delta}\right)\right)\right)+\cos \left(\theta_{R n}-\theta_{\Delta}\right)\right)
$$

azimuth of observation point in $n$-th antenna:

$$
\theta_{n}=\arctan \frac{\left|\mathbf{R}_{n}\right| \sin \left(\theta_{R n}\right)+|\Delta| \sin \left(\theta_{\Delta}\right)}{\left|\mathbf{R}_{n}\right| \cos \left(\theta_{R n}\right)+|\Delta| \cos \left(\theta_{\Delta}\right)} .
$$

Search of $\theta_{n}, \Delta_{n}$ expressions is possible with precise definition of the PAA polygon parameters $\mathbf{R}_{n}\left(\left|\mathbf{R}_{n}\right| ; \theta_{R n}\right)$. In case of irregular polygonal structure, obtaining of its parameters which depend on $d_{i j}$, antenna and phase center positions is a hard analytical task. This task can be solved by empirical methods. Another way is predetermination of these parameters in the condition of preserving polygonal structure. 
For regular polygonal structure with a side $d$, the task of obtaining of its parameters is much easier, because $\mathbf{R}_{n}$ is the bisecting line of each polygon corner and $\left|\mathbf{R}_{n}\right|$ is the radius of polygon escribed circle, where $n \in[1 ; N], n \in \mathbf{Z}, N$ - polygon sides quantity. Then:

$$
\begin{gathered}
\left|\mathbf{R}_{n}\right|=\frac{d}{2 \sin (\pi / N)} ; \\
\theta_{R n}=\frac{\pi}{N}(2 n-1) .
\end{gathered}
$$

Expression for spatial shifts between elements of antenna array with nonlinear structures, for example, parabola, hyperbola, can be obtained in the same way as for PAA.

\subsection{Focused arrays radiation pattern}

Radiation pattern of the near-field or the intermediate-field of FAA has the following features compared with common antenna arrays focused at the far-field zone (FFZ):

- $\quad$ signal attenuation by propagation $L(n, R)=4 \pi R^{2}(n) / \lambda^{2}$ is different from each FAA $n$-th antenna element due to different distance to observation point $R(n)$;

- $\quad$ each $n$-th element of FAA radiates signals to observation point at different angles;

- each element of FAA has the radiation pattern $\dot{f}_{n}(\theta(n), R)$ that depends on the distance;

- $\quad$ assumed that the near field of FAA doesn't include its elements reactive field;

- main lobe, side lobes, grating lobes that are expressed in angular values are being transformed to main, side and grating focal areas that are expressed in values of width, length and height, that causes the problem of transforming from polar (spherical) to Cartesian coordinates.

Then the radiation patterns of FAA which consist of phase shifters and delay lines according to a common theory (Hansen, 2009) are defined respectively as:

$$
\begin{gathered}
\dot{F}(\theta, R)=\sum_{n} \frac{\dot{f}_{n}(\theta(n), R)}{L(n, R)} e^{j(k(\omega) \Delta(n)-\Delta \Phi(n))} ; \\
\dot{F}(\theta, R)=\sum_{n} \frac{\dot{f}_{n}(\theta(n), R)}{L(n, R)} e^{j(\omega(\Delta(n) / c-\Delta T(n)))} ;
\end{gathered}
$$

where $I(n)$ - $n$-th antenna element excitation amplitude;

$\Delta \varphi(n)=k \Delta(n)$ - the phase shift between phase center and the $n$-th antenna element;

$\Delta t(n)=\Delta(n) / c$ - time delay between the phase center and the $n$-th antenna element;

$\Delta \Phi(n), \Delta \mathrm{T}(n)$ - the phase and time distribution of $n$-th antenna element excitation;

$\omega$ - circular frequency;

$k=2 \Pi / \lambda=\omega / c$ - coefficient of propagation;

$\lambda$ - signal wavelength;

$c$ - signal propagation speed in the environment.

If FAA is excited by wideband signal with multicomponent spectrum, for example, pulse signals, definition of its radiation pattern cannot be completed without taking into account the spectral structure of the signal $\dot{A}(j \omega), \omega \in\left[\omega_{\min } ; \omega_{\max }\right]$ - signal circular frequency band. 
Therefore, this expression can be described in form of inverse Fourier transformation of radiation patterns at each spectrum component with their amplitude and phase as follows:

$$
\begin{gathered}
\dot{F}(\theta, R)=\sum_{\omega} \dot{A}(j \omega) \sum_{n} \frac{\dot{f}_{n}(\theta(n), R)}{L(n, R)} e^{j(k(\omega) \Delta(n)-\Delta \Phi(n, \omega))} ; \\
\dot{F}(\theta, R)=\sum_{\omega} \dot{A}(j \omega) \sum_{n} \frac{\dot{f}_{n}(\theta(n), R)}{L(n, R)} e^{j(\omega(\Delta(n) / v-\Delta T(n)))} ;
\end{gathered}
$$

The concept of FAA excitation by wideband signal is introduced in papers $(\mathrm{Wu}, 1985$; Kremer, 1984) and was used by several authors (Ishimaru et al.,2007; Hussain, 2004; Malyuskin \& Fusco, 2009) to improve FAA performance, but they had not disclosed the nature of this method. Expression (17a) is used for a broadband signal in the time sense and (17b) in the space-time sense (Kremer, 1984).

For example, the expression for radiation pattern of focused LAESR can be written as:

$$
\dot{F}(\theta, R)=\sum_{\omega} \dot{A}(j \omega) \sum_{n} \frac{\dot{f}_{n}(\theta(n), R)}{L(n, R)} e^{j\left(k(\omega) n d\left(\cos (\theta) \tan \left(0.5 \operatorname{arccot}\left(\frac{R_{F}}{d n \cos (\theta)}-\tan (\theta)\right)\right)-\sin \theta\right)-\Delta \Phi(n, \omega)\right)},
$$

where $\Delta \Phi(n, \omega)=k(\omega) n d\left(\cos \left(\theta_{F}\right) \tan \left(0.5 \operatorname{arccot}\left(\frac{R_{F}}{d n \cos \left(\theta_{F}\right)}-\tan \left(\theta_{F}\right)\right)\right)-\sin \theta_{F}\right)$;

$R_{F}, \theta_{F}$ - coordinates of focus point.

Radiation patterns intersection in the azimuth plane of LAESR of nine omnidirectional in azimuth plane elements with $d=\lambda / 2$ and octagonal structure PAA of patch antenna elements with radiation pattern in a form of cardioid which are radiating in the center of polygon with $d=2 \lambda$ without taking into account signal attenuation by the propagation are shown in Fig. 3(a) and Fig. 3(b) respectively, where the signal level is in dB.

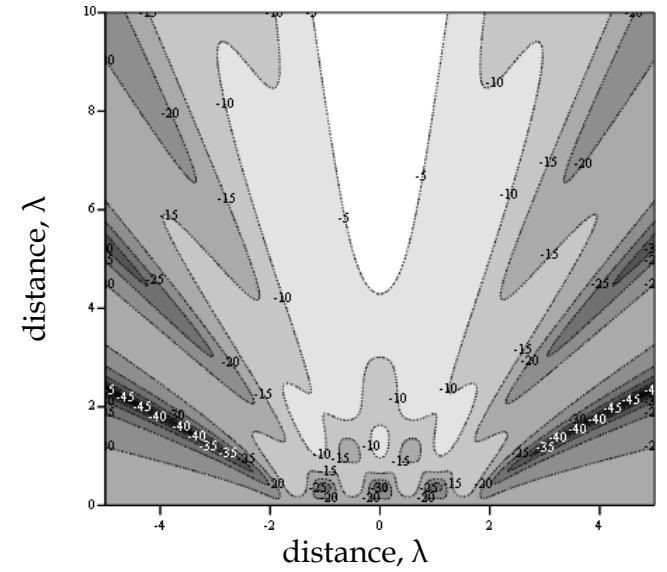

(a)

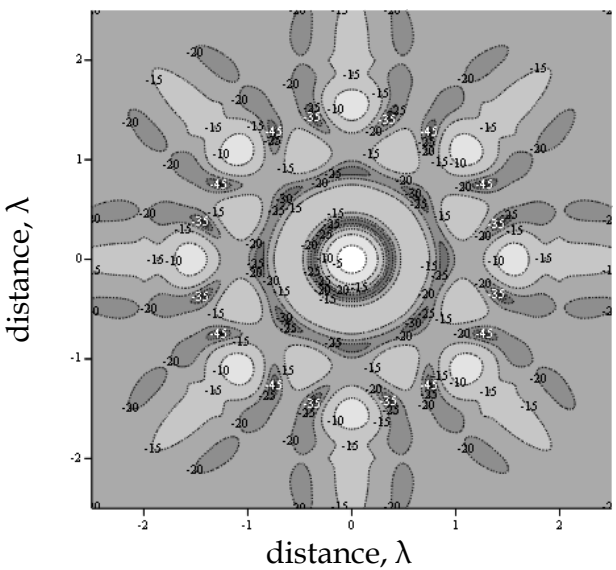

(b)

Fig. 3. Radiation patterns intersection in the azimuth plane of NFZ and IFZ of LAESR, PAA 
According to Fig.3 and sources (Fenn, 2007; Hansen, 1964; Graham, 1983), the features of radiation patterns by distance are the same as by angle coordinates. That is the level and the amount of mainlobe and sidelobes proportionally to the level and the amount of main focal area and side focal areas. Thus the methods of radiation pattern synthesis (Hansen,2009) in NFZ, IFZ and FFZ are similar.

\subsection{Focusing properties of antenna arrays}

According to a common theory (Hansen, 1964), focusing - the process of compensation of components of phase shifts between antenna elements and phase center of FAA, that depends on the distance in focus coordinates $R_{\mathrm{F}}, \theta_{\mathrm{F}}$. Otherwise focusing is the process of inversion of the Fresnel and the Fraunhofer diffraction fields. Thereby FAA achieves a high directivity in its NFZ or IFZ and loses directivity in its FFZ.

The important factor is that the focus takes place exclusively in NFZ or IFZ (Hansen, 1964) limited for LAESR by the hyperfocal distance from its phase centre $R_{H F}<D^{2} \cos ^{2}(\theta) / \lambda$, where $D$ - the largest FAA dimensions in the plane where NFZ or IFZ is considered. Depth of focus and directivity are important parameters of FAA that describe its focusing or directional signal transmission properties.

According to (Hansen, 1964), depth of focus - the size of focal area in the dimension of distance $R \in\left[R_{\text {near }}, R_{\text {far }}\right]$. Expressions for $R_{\text {near }}$ and $R_{\text {far }}$ (Hansen, 1964), where the level of focused LAESR radiation patterns by distance becomes $-0,1 \mathrm{~dB}$ are obtained by methods of theory of geometrical optics and are defined as:

$$
\begin{gathered}
R_{\text {near }}=\frac{D^{2} / \lambda}{1+D^{2} / \lambda R_{F}\left(\theta_{F}\right)} \\
R_{\text {far }}=\left\{\begin{array}{cc}
\frac{D^{2} / \lambda}{-1+D^{2} / \lambda R_{F}\left(\theta_{F}\right)}, & R_{F}\left(\theta_{F}\right)<\frac{D^{2} \cos ^{2} \theta_{F}}{\lambda}=R_{H F} \\
\infty, & R_{F}\left(\theta_{F}\right) \geq \frac{D^{2} \cos ^{2} \theta_{F}}{\lambda}=R_{H F} .
\end{array}\right.
\end{gathered}
$$

Graham (Graham, 1983) had studied the axial field radiation pattern synthesis for apertures focused in the Fresnel region with Fresnel approximation, and had obtained the equations for depth of focus $\Delta R$, minima $R_{\min }$ and maxima $R_{\max }$ of field location. These equations can be used for focused LAESR and can be written for different angles of focus point in azimuth plane, respectively as:

$$
\begin{gathered}
\Delta R=\frac{7 \lambda\left(R_{F} \cos \left(\theta_{F}\right)\right)^{2}}{D^{2}-\left(3.5 \lambda R_{F} \cos \left(\theta_{F}\right) / D\right)^{2}} ; \\
R_{\min }=\frac{R_{F} \cos \left(\theta_{F}\right)}{1-8 n \lambda R_{F} \cos \left(\theta_{F}\right) / D^{2}}, \text { for } n<\frac{D^{2}}{8 \lambda R_{F} \cos \left(\theta_{F}\right)}, n \in \mathbf{Z} ; \\
R_{\max }=\frac{R_{F} \cos \left(\theta_{F}\right)}{1-8(n+0.5) \lambda R_{F} \cos \left(\theta_{F}\right) / D^{2}}, \text { for } n<\frac{D^{2}}{8 \lambda R_{F} \cos \left(\theta_{F}\right)}-0.5, n \in \mathbf{Z} ;
\end{gathered}
$$


Equations (20), (21), (22) are exact only in IFZ.

The exact equations for LAESR parameters $\Delta R, R_{\min }$ and $R_{\max }$ in NFZ can be obtained when the problem of finding an exact expression for its radiation pattern is solved. Equation for LAESR radiation pattern in azimuth plane can be written as follows:

$$
F(\theta, R)=e^{-j \beta R} \int_{v_{L S E}}^{v_{R S E}} e^{j \beta R\left(\frac{\cos (\theta)}{\cos (v-\theta)}\right)} \mathrm{d} v
$$

where $v_{L S E}=\operatorname{arccot}\left(\frac{R}{-d N \cos (\theta)}+\tan (\theta)\right), v_{R S E}=\operatorname{arccot}\left(\frac{R}{d N \cos (\theta)}-\tan (\theta)\right)$,

For further description the parameters of FAA radiation patterns equations (20), (21), are used since the expression (23) has no solution in the form of simple function.

Radiation patterns intersection in the azimuth plane of LAESR of twenty one patch antenna elements focused in polar coordinates at $R_{F}=50 \lambda, \theta_{F}=0^{\circ}$ with $d=\lambda$ and phase centre in eleventh element with and without taking into account signal attenuation by the propagation are shown in Fig.5(a) and Fig.5(b) respectively, where the signal level is in dB. Axial radiation patterns on azimuth $\theta_{F}=0^{\circ}$ of this LAESR focused at $R_{F}=50 \lambda$ with and without taking into account signal attenuation by the propagation are shown in Fig.6.

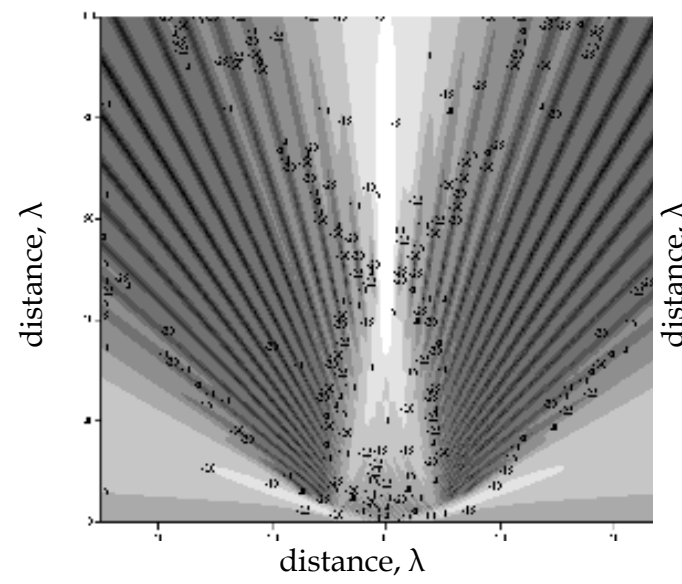

(a)

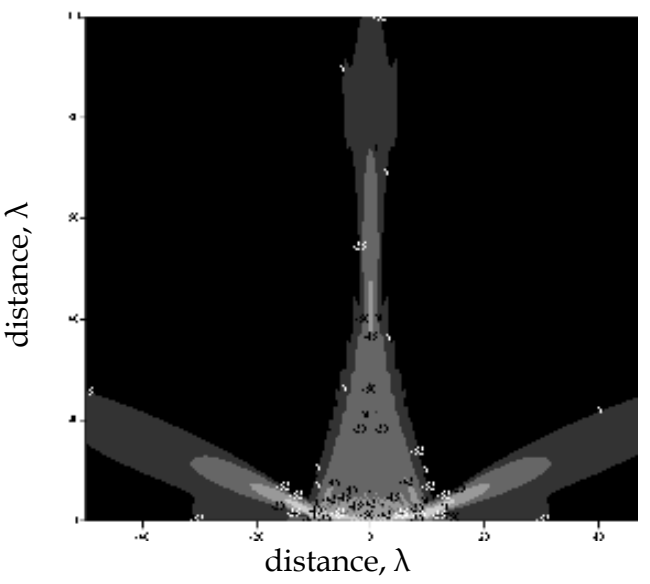

(b)

Fig. 5. Radiation patterns intersection in the azimuth plane of LAESR

Fig.5, Fig.6 and source (Hansen, 1964) shows that the focal area or a certain segment of distance $R \in\left[0, R_{F}\right]$ where radiated power distribution is uniform can be the result of focusing process.

The level of radiation pattern in FFZ becomes the level of radiation pattern in point of NFZ or IFZ of LAESR focused on FFZ as the result of focusing on this point according to the fact that focusing is the process of inversion of the Fresnel and the Fraunhofer diffraction fields, as shown on Fig.7.

From Fig.7 and the materials (Hansen, 1964) it can be concluded that the minimum signal level in FFZ of FAA can be obtained since FAA is focused in points of minimum signal level 
when it is focused in FFZ. These focus points are calculated from (21). The closer the focus point is placed then the weaker the level of signal in the FFZ is.

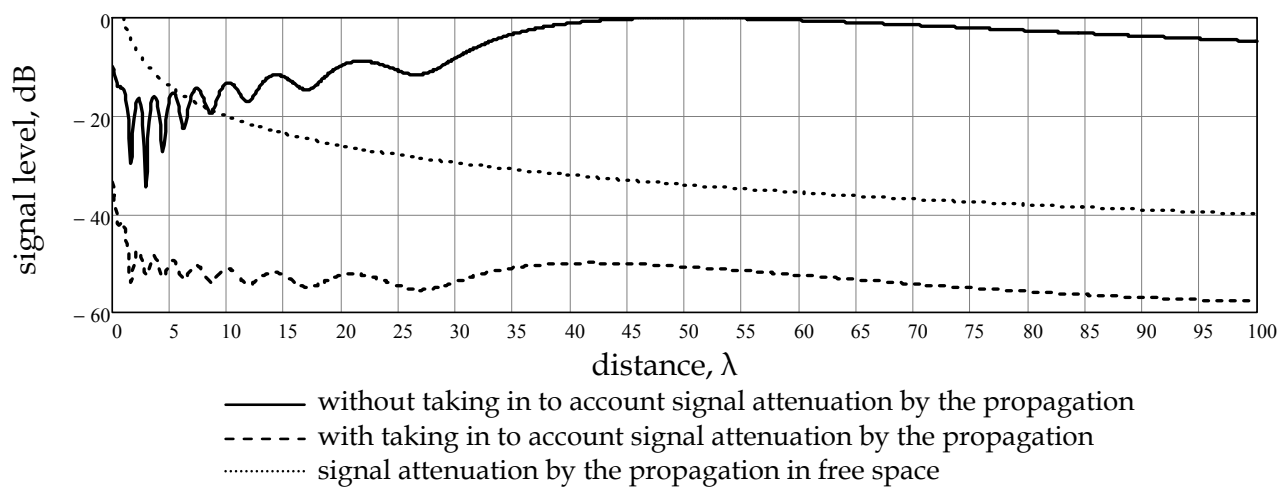

Fig. 6. Axial radiation patterns on azimuth $\theta_{F}=0^{\circ}$ of focused LAESR

Radiation patterns intersection in the azimuth plane of octagonal PAA of patch antenna elements which are radiating inside the structure and focused in the center of polygon with $d=3 \lambda, \lambda=2 \mathrm{~m}$ with and without taking into account signal attenuation by the propagation are shown in Fig.8(a) and Fig.8(b) respectively, where the signal level is in $\mathrm{dB}$.

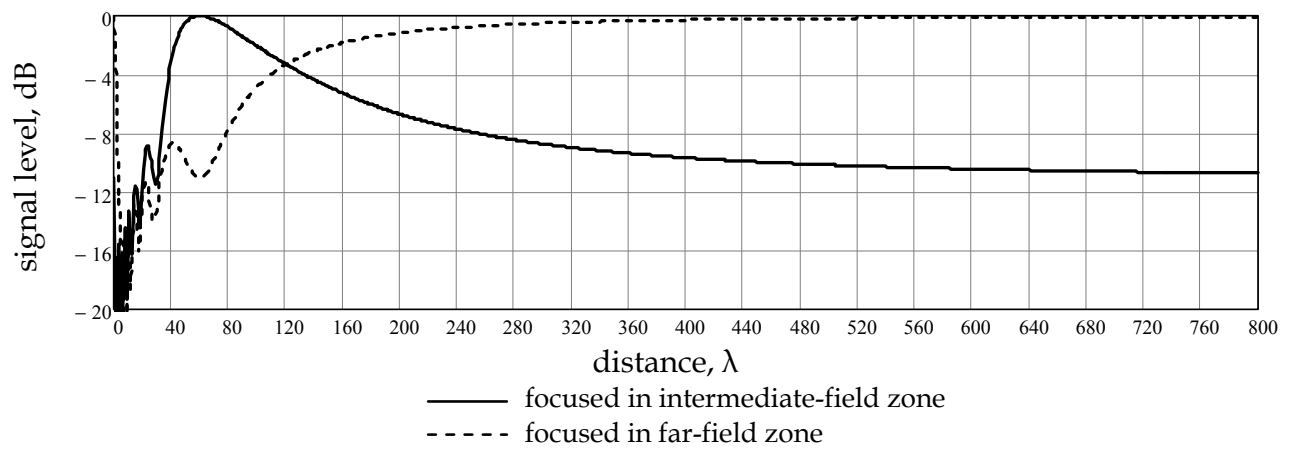

Fig. 7. Axial radiation patterns of LAESR focused in NFZ and FFZ

For antenna array of polygonal structures where all elements are radiating inside the polygon, radiation pattern is described as field in NFZ or IFZ of LAESR. Therefore, according to Fig.8 and the paper (Mazurenko \& Yakornov, 2010) generally focal area has the form of ellipse, and for regular polygon array structures the form of circle with depth of focus $\Delta R \approx \lambda / 3$.

The focus point of antenna array of regular polygonal structure with $N<7, d<\lambda$ where $\left|\mathbf{R}_{n}\right|<\lambda$ is located in its reactive field zone that can be avoided by increasing the number of antenna elements $N$ in the condition $\left|\mathbf{R}_{n}\right|>\lambda$ or increasing the element spacing $d>>\lambda$. Radiation of wideband signals by array or using the directional antennas is required for grating focal areas suppression since $d>>\lambda$. The method of radiation of wideband signals for grating focal areas suppression will be reviewed in the next section. The preliminary simulation result of this method in form of radiation pattern intersection in the azimuth 
plane of octagonal PAA of patch antenna elements which are radiating inside the structure periodic pulse signal with bandwidth $\Delta f=300 \mathrm{MHz}$, central frequency $f_{C}=150 \mathrm{MHz}$, period $T=33.3 \mathrm{~ns}$, pulse duration $\tau_{\mathrm{p}}=6.6 \mathrm{~ns}$ and focused in the center of polygon with $d=4 \lambda, \lambda=2$ $\mathrm{m}$ with taking into account signal attenuation by the propagation is shown in Fig. 9.

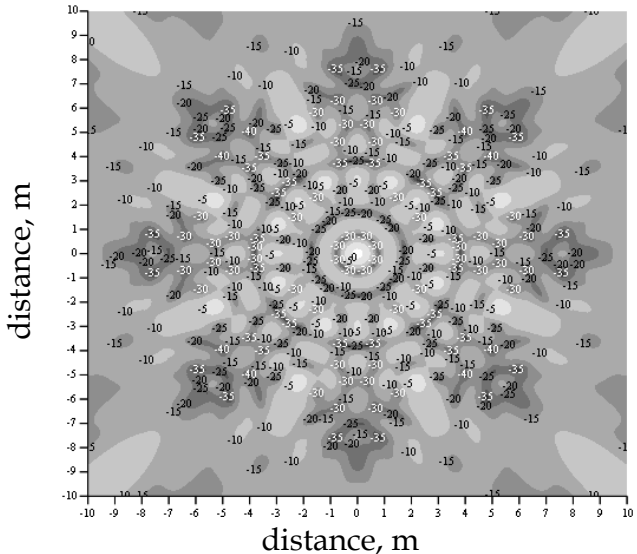

(a)

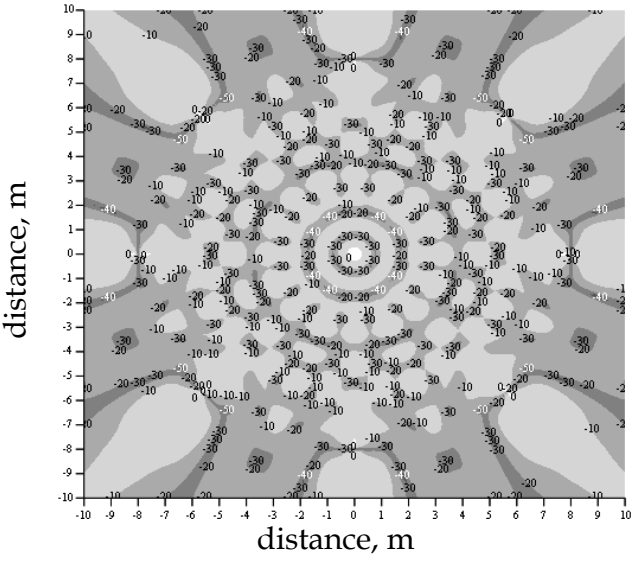

(b)

Fig. 8. Radiation patterns intersection in the azimuth plane of octagonal PAA

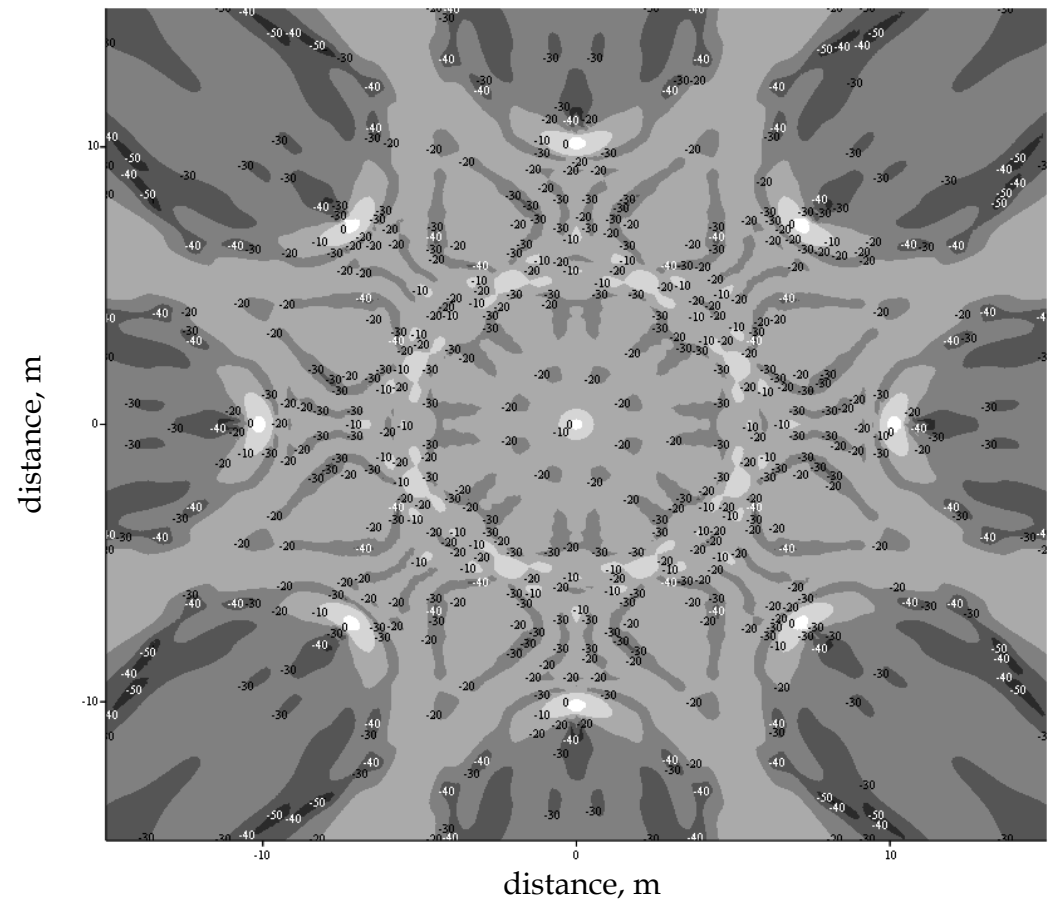

Fig. 9. Radiation patterns intersection in the azimuth plane of octagonal PAA 
According to study results presented in the papers (Hansen, 1964; Polk, 1956), the directivity of focused linear antenna array in NFZ or IFZ cannot exceed directivity in its FFZ since assumed that the focusing is the process of inversion of Fresnel and Fraunhofer diffraction fields. Thus the methods of calculating the angular directivity of FAA are equal to methods of calculating the directivity of common antenna arrays in the FFZ (Hansen, 2009).

The polygonal structure array directivity is defined by degree of radiation inside it.

In common sense antenna directivity is relative to angular selectivity. For selectivity by distance the determination of antenna directivity is a complex task due to infinity of values of coordinate by distance. Thereby the directivity by distance can be determined only as angular directivity in focused point to angular directivity in FFZ ratio. The angular directivity as a function of distance with Fresnel approximation for focused apertures can be obtained from paper (Polk, 1956).

The overall FAA directivity as a multiplication of directivity by distance and angle coordinates can be obtained.

The overall antenna gain, obtained by focusing process cannot be calculated without taking into account signal attenuation by the propagation.

Thereby appliance of FAA is limited by distance $R_{M D}$ due to signal attenuation by propagation that can be described as:

$$
R_{M D} \rightarrow L\left(R_{M D}\right) \leq G_{A S} G_{A E} S I N R,
$$

where $G_{A S}$ - gain of FAA;

$G_{A E}$ - gain of FAA antenna element;

SINR - necessary signal to interference and noise ratio at the appropriate detector.

The FAA ability to separate signals by distance as by angular coordinates can be concluded according to the materials of this section.

\section{Focused Antenna Arrays directivity improving methods}

Improving of FAA directivity is increasing its spatial resolution by distance and angular coordinates and increasing the hyperfocal distance. The first attempts of FAA directivity improvements are made in the papers (Karimkashi \& Kishk, 2009; Hussain \& Al-Zayed, 2008), but the authors used common methods of radiation pattern synthesis in the FFZ and in a case of array excitation by wideband signal (Hussain, 2004, 2008) the nature of improvement had not been disclosed.

According to the materials of previous section and the papers mentioned before in this section, FAA directivity can be improved by increasing element spacing jointly with using directed array elements (Hansen, 2009) or exciting wideband signal jointly with increasing array elements sparsity (Hussain \& Al-Zayed, 2008). Also this task can be solved by using common methods of optimal radiation pattern synthesis for FFZ (Hansen, 2009).

Application of common methods of pattern synthesis by special amplitude-phase distributions (APD) of excited signal or spatial distributions of array elements despite the papers (Karimkashi \& Kishk, 2009; Hussain \& Al-Zayed, 2008) is not so effective for FAA as for conventional antenna arrays. Low efficiency of these methods may be linked to the fact that they are created for linear distribution of phase shifts between elements of array and the process of focusing in NFZ or IFZ based on creation of non-linear distribution. Thus application of specific APD (Hansen, 2009) created for far-field pattern synthesis, wideband signal radiation with linear distribution of spectrum components, for example, pulse signals 
(Hussain \& Al-Zayed, 2008) for FAA is leading to its partial defocusing in NFZ or IFZ. This defocusing occurs due to ignoring of phase shifts components that depend on distance when common APD and amplitude-frequency characteristic (AFC) of radiated signal had been created. Modification of specific antenna array APD or AFC of wideband signals, which is radiated by arrays is the solution for defocusing problem.

Use the concept of spectrum of spatial frequencies (SSF) for finding a solution of this defocusing problem. The SSF concept is presented from different sides in the books (Korostelev, 1987; Kremer, 1984). Also the SSF concept helps to reveal the nature of wideband signal excitation of FAA to improve its directivity.

The essence of SSF concept is the similarity of use of the inverse Fourier transformation for the synthesis signal in time domain with a limited frequency spectrum and radiation pattern in the spatial region using spatial frequency spectrum which is essentially the APD of array excitation.

Radiation patterns of antenna array of two elements excited by the wideband signal with $m$ components frequency spectrum and $m$-elements antenna array excited by the narrowband signal are equivalent that can be concluded from the use of the SSF concept, where space frequency $\omega_{\mathrm{S}}=2 \Pi d_{0} n / \lambda_{m}=2 \Pi d_{0} n f_{m} / c=2 \Pi d_{0} n f_{0} m / c, d_{0}=l \lambda_{0}, l$ - normalized array spacing in wavelength, $f_{0}=c / d_{0}, f_{m}=f_{0} m, c$ - signal propagation speed in the environment. Thereby each frequency spectrum component $f_{m}$ except $f_{0}$ of excited signal creates a SSF component or a virtual antenna array element located from its phase centre at distance $d_{m}=d_{0} m$ that can be assumed. So SSF can be linked with AFC of radiated signal.

When array is excited by the signal with spectrum components $f_{m}<f_{0}, m=f_{m} / f_{0}<1$, the grating lobes and the side lobes are suppressing, the main lobe becomes wider. When array is excited by the signal with spectrum components $f_{m}>f_{0}, m=f_{m} / f_{0}>1$, the grating lobes and the side lobes are not suppressing, the main lobe becomes narrower. Similar result is obtained in the paper (Hussain \& Al-Zayed, 2008).

Thus for increasing the directivity of antenna array by the method of increasing array elements sparsity jointly with wideband signal excitation is necessary to enhance the array element spacing $d_{0}=l \lambda_{0}$ with $l>1$ and to excite array by the signal with spectrum components $f_{m}<f_{0}, m=f_{m} / f_{0}<1$. The directivity increasing is much effective when created SSF components are located from its phase centre at distance $d_{m}=p \lambda_{0} / 2, p \in \mathbf{Z}$ or $f_{m}=p f_{0} / 2 l$, $p / 2 l<2$. So this method is effective when radio impulse signal is radiated by FAA. For example, excitation LAESR with element spacing $d_{0}=4 \lambda_{0}$ by the periodic pulse signal with bandwidth $\Delta f=300 \mathrm{MHz}$, central frequency $f_{C}=f_{0}=150 \mathrm{MHz}$, period $T=33.3 \mathrm{~ns}$, pulse duration $\tau_{\mathrm{p}}=6.6 \mathrm{~ns} 4$ times increases directivity and 16 times increases hyperfocal distance in comparison to the array with element spacing $d_{0}=\lambda_{0}$ and narrowband signal excitation. Radiation patterns intersection in the azimuth plane of LAESR of 11 patch antennas focused in polar coordinates at $R_{F}=50 \mathrm{~m}, \theta_{F}=0^{\circ}$ with $d_{0}=4 \lambda_{0}, \lambda_{0}=0.5 \mathrm{~m}$ excited by narrowband and by wideband signal are shown in Fig.10(a) and Fig.10(b) respectively.

For increasing the hyperfocal distance by the method of wideband signal excitation is necessary to excite array by the wideband or ultrawideband pulse signal with spectrum components $f_{m}>f_{0}, m=f_{m} / f_{0}>1$. The hyperfocal distance is linearly dependent on frequency band, so $R_{H F m}=m R_{H F 0}$. Using of this method causes the grating focal area generating and the side focal area rising, so directivity by angular coordinate is decreased, but directivity by distance is increased, that are dependent on AFC of radiated signal as in previous method. For increasing the directivity of antenna array by the method of using special APD or AFC is necessary to modify APD or AFC created for FFZ beamforming (original AFC can be 
taken from previous methods) by the way of transformation of SSF distributions from the linear $A\left(n \omega_{s}\right)$ to nonlinear form with Fresnel approximation $A\left(\left[n-n^{2} / 2 R_{F}\right] \omega_{S}\right)$, where $n$ number of array elements; $R_{F}$ - focusing distance. This transformation is performed by transferring the amplitude distribution of SSF with nonlinear distribution to SSF with linear distribution of its components, when the FAA elements are uniformly spaced, because SSF components distribution is equal to FAA elements spatial location distribution or AFD of signal radiated by the FAA.

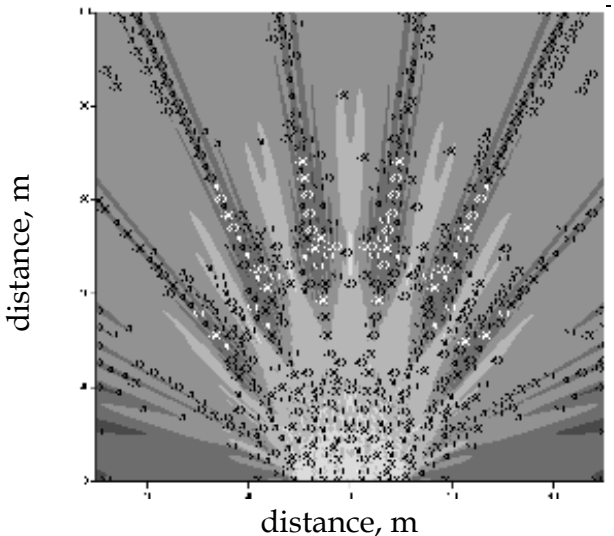

(a)

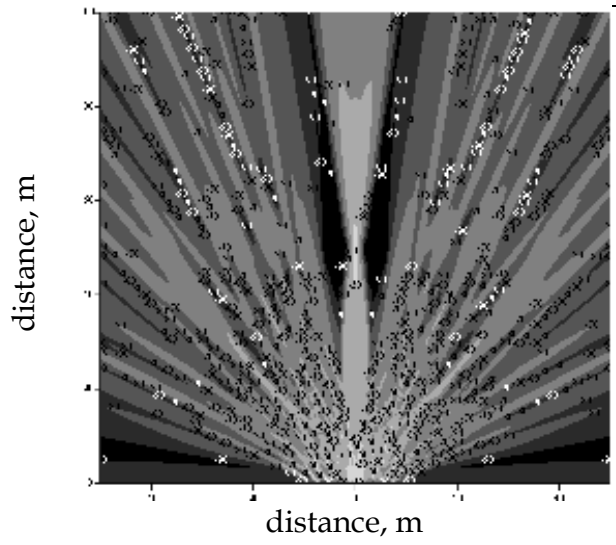

(b)

Fig. 10. Radiation patterns intersection in the azimuth plane of focused LAESR

For example, the modification of Dolph-Chebyshev APD leads to APD of the same type, but for radiation pattern with higher side lobe level and narrower main lobe (Fig. 11), which essentially gives the best result. So, using Dolph-Chebyshev APD causes the defocusing versus side focal area suppressing in all cases as for FFZ beamforming.

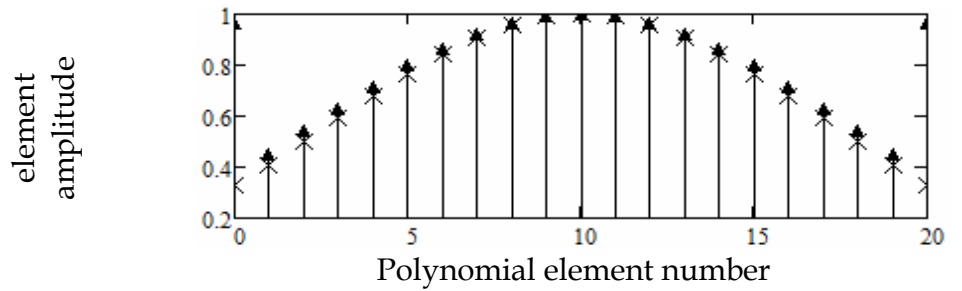

$\Delta$ Modified Dolph-Chebyshev APD XOriginal Dolph-Chebyshev APD

Fig. 11. Modification of Dolph-Chebyshev APD for LAESR of 21 antenna elements

Modification of AFC of wideband pulse signal emitted by FAA is done in same way and causes transformation of all parameters of radiated signal. This fact allows to consider the problem of optimal signals for high directivity of antenna arrays achieving. A comparison of radiation patterns by distance of LAESR which radiating wideband periodic radio impulse with modified and common AFC is shown in Fig.12(a) and comparison of radiation patterns by distance of LAESR with modified and common Dolph-Chebyshev APD which radiating wideband periodic radio impulse is shown in Fig.12(b). Fig.12 shows that this modification is effective, thus the defocusing is present indeed. 
These modifications are effective only in NFZ. This method can be used only for linear FAA. The using of the methods of radiation pattern synthesis which are developed using SSF concept and studied by the authors of this chapter in this section is necessary for improve FAA performance according to materials mentioned before.

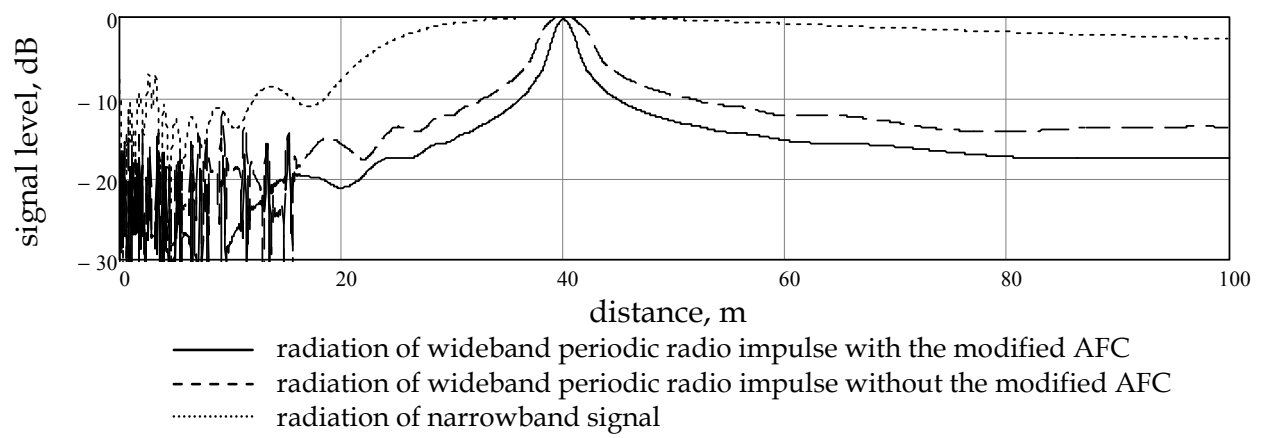

(a)

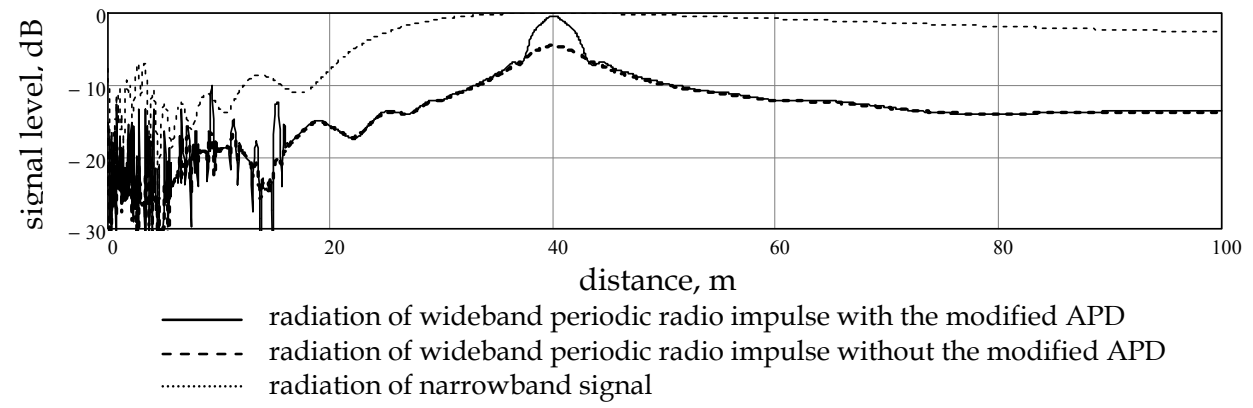

(b)

Fig.12. Axial radiation patterns of LAESR of 21 antenna elements excited by special signals

\section{Focused Antenna Arrays in telecommunications engineering}

Radio-frequency resource utilization efficiency in the spatial area is not enough because the spatial selectivity is limited by two dimensions: the azimuth and the elevation. This problem can be solved by use of antenna systems with controlled 3-dimensional, including distance, signal spatial division or FAA, which form operational area in their NFZ and IFZ.

Modern technics of focused antennas are presented by arrays (Buffi et al., 2009; Fenn, 2007; Malyuskin \& Fusco, 2009), Fresnel zone plate and lens antennas (Garret \& Wiltse, 1990; Herben, 1999; Hristov, 2004; Karimkashi \& Kishik, 2008; Reid \& Smith, 2009; Wang et al., 2007) and other (Rudolph \& Grbic, 2008; Molotkov et al., 2009). The arrays and single radiators of these focused antennas can be used for 3-dimensional directional signal transmission.

Applicability of the antennas mentioned before for short range fixed or mobile communication is provided by its capability to perform the controlled 3-dimensional 
spatial signal division in necessary operational area of wireless telecommunication system.

According to the paper (Mazurenko \& Yakornov, 2010), realization of the given FAA for achieving operation zone dimensions $R_{O A}=0.1 \ldots 1 \mathrm{~km}$ and focal area with depth of focus $\Delta R$ $=10 \ldots 100 \mathrm{~m}$ are limited by the hyperfocal distance. For FAA element spacing $d_{0}=l \lambda_{0}$ the operation area dimension $R_{O A}$ and array parameters are related by expression

$$
R_{O A}<(N-1)^{2} l^{2} \lambda_{0}
$$

According to eq. (25) and materials of previous sections, FAA structures for some frequency ranges are:

- $f<30 \mathrm{MHz}$ - element quantity $N<10$, rarefied antenna array of polygonal structure with electrically small antenna elements.

- $f \in[30 \mathrm{MHz} ; 30 \mathrm{GHz}]$ - element quantity $N \in[10 ; 100]$, rarefied antenna array of polygonal or linear structure with directional array elements and grating focal areas suppression methods application.

- $f>30 \mathrm{GHz}$ - linear or planar antenna array with element quantity $N>100$ or equivalent length continuous radiator, aperture, reflector.

Considering all achievements in FAA directivity improving and real condition of signal propagation the following antenna systems can be created:

- PAA with operation zone in the area inside structure of polygon. Radiation pattern of PAA is characterized by one focal area of circular form with power gain $<10 \mathrm{~dB}$ if maximum operational zone dimension not exceed $100 \mathrm{~m}$, as shown on Fig.13.

- $\quad$ LAESR with operation zone in the area of its NFZ and IFZ. Radiation pattern of LAESR is characterized by one focal area of elliptical form with power gain 10-20 dB if maximum operational zone dimension not exceed $100 \mathrm{~m}$, as shown on Fig.14, and by almost uniformly distributed power over the area with width equal to array maximum dimensions and length equal to focus point distance with power fluctuations $<5 \mathrm{~dB}$ if maximum operational zone dimension within the limits $R_{O A} \in[100 \mathrm{~m} ; 1000 \mathrm{~m}]$, as shown on Fig.15.

Radiation patterns intersection in the azimuth plane of octagonal PAA of patch antennas with $d=10 \lambda, \lambda=4 \mathrm{~m}$ which are radiating inside the structure periodic radio impulse signal with $\Delta f=150 \mathrm{MHz}, f_{C}=75 \mathrm{MHz}, T=173.3 \mathrm{~ns}$ and $\tau_{\mathrm{p}}=13.3 \mathrm{~ns}$ with taking into account signal attenuation by the propagation, where array focused in point with coordinates $R_{F}=0$ $\mathrm{m}, \theta_{F}=0^{\circ}, R_{F}=15 \mathrm{~m}, \theta_{F}=45^{\circ}$ relatively to the center of polygon are shown in Fig.13(a) and Fig.13(b) respectively, where the signal level is in $\mathrm{dB}$.

Radiation patterns intersection in the azimuth plane of LAESR of 401 patch antennas with $d$ $=\lambda$ and $\lambda=5 \mathrm{~mm}$ or equivalent reflector with linear dimension $D=2 \mathrm{~m}$ with taking into account signal attenuation by the propagation, where array radiated narrowband signal in focal points with coordinates $R_{F}=100 \mathrm{~m}, \theta_{F}=0^{\circ}$ and $R_{F}=100 \mathrm{~m}, \theta_{F}=30^{\circ}$ are shown in Fig.14(a) and Fig.14(b) respectively, where the signal level is in $\mathrm{dB}$.

Radiation patterns intersection in the azimuth plane of LAESR of 401 patch antennas with $d$ $=\lambda$ and $\lambda=5 \mathrm{~mm}$ or equivalent reflector with linear dimension $D=2 \mathrm{~m}$ with taking into account signal attenuation by the propagation, where array radiated narrowband signal in focal points with coordinates $R_{F}=800 \mathrm{~m}, \theta_{F}=0^{\circ}$ and $R_{F}=800 \mathrm{~m}, \theta_{F}=30^{\circ}$ are shown in Fig.15(a) and Fig.15(b) respectively, where the signal level is in $\mathrm{dB}$. 


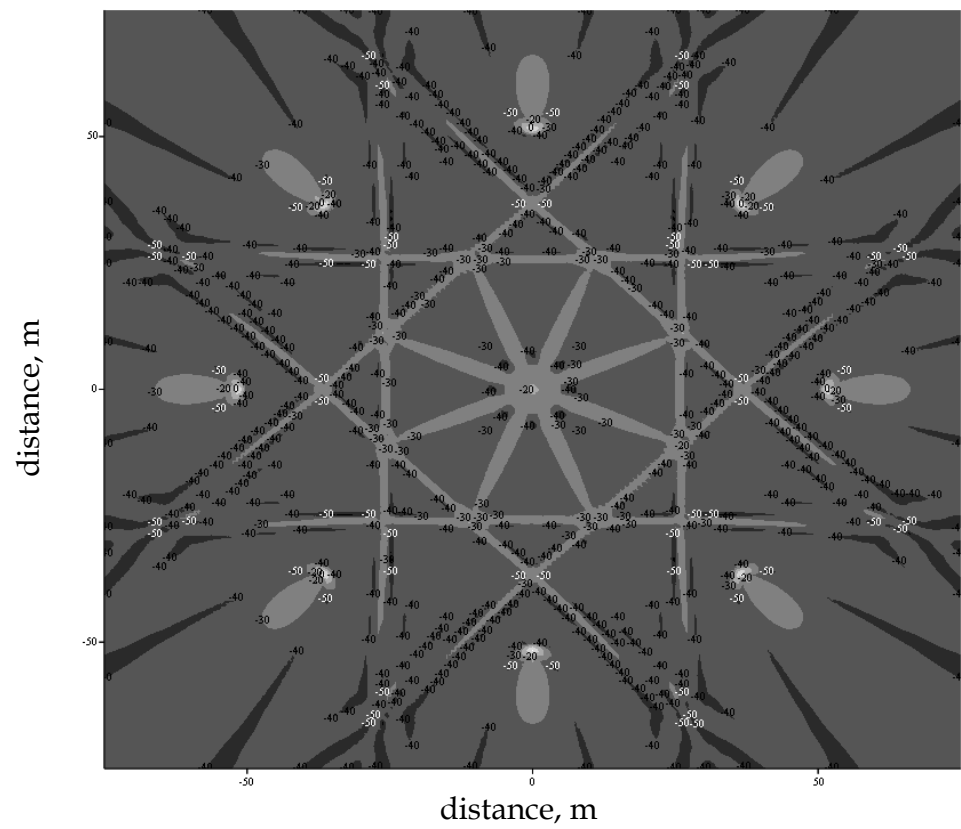

a)

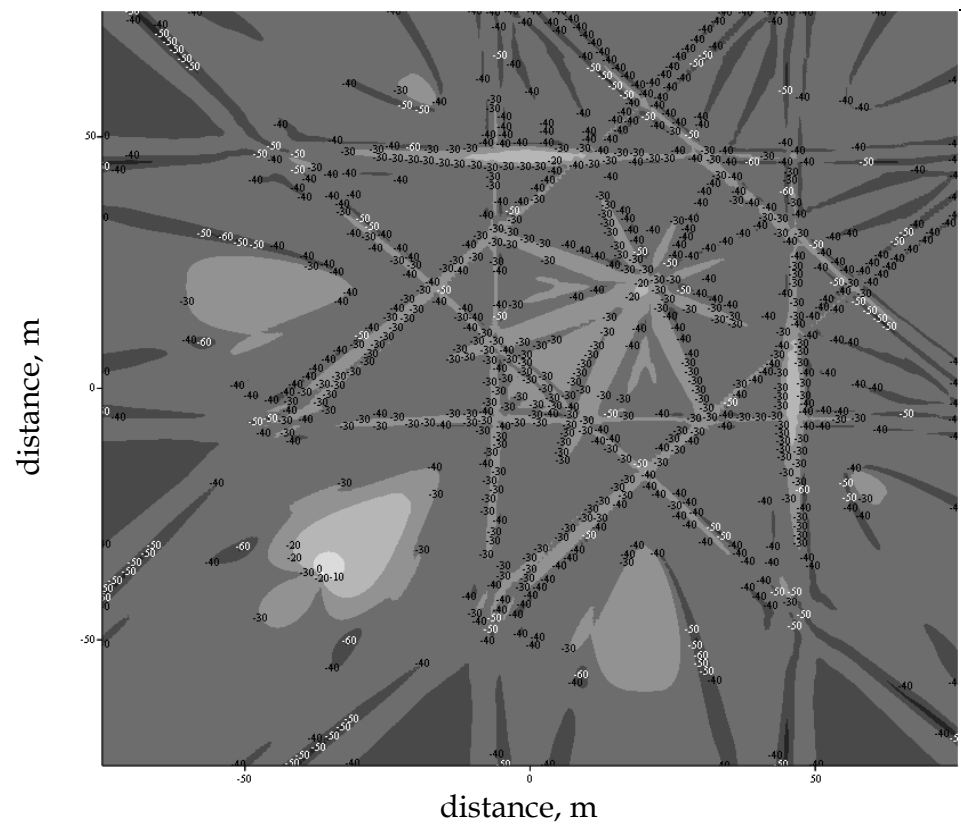

b)

Fig. 13. Radiation patterns intersection in the azimuth plane of octagonal PAA 


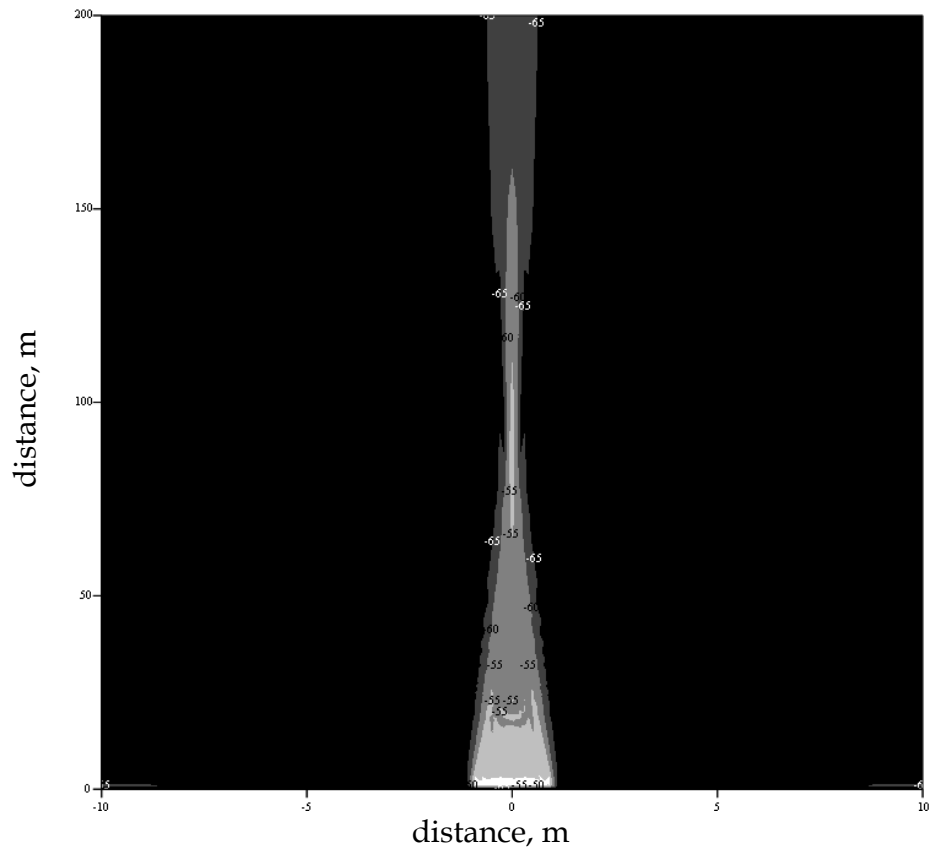

(a)

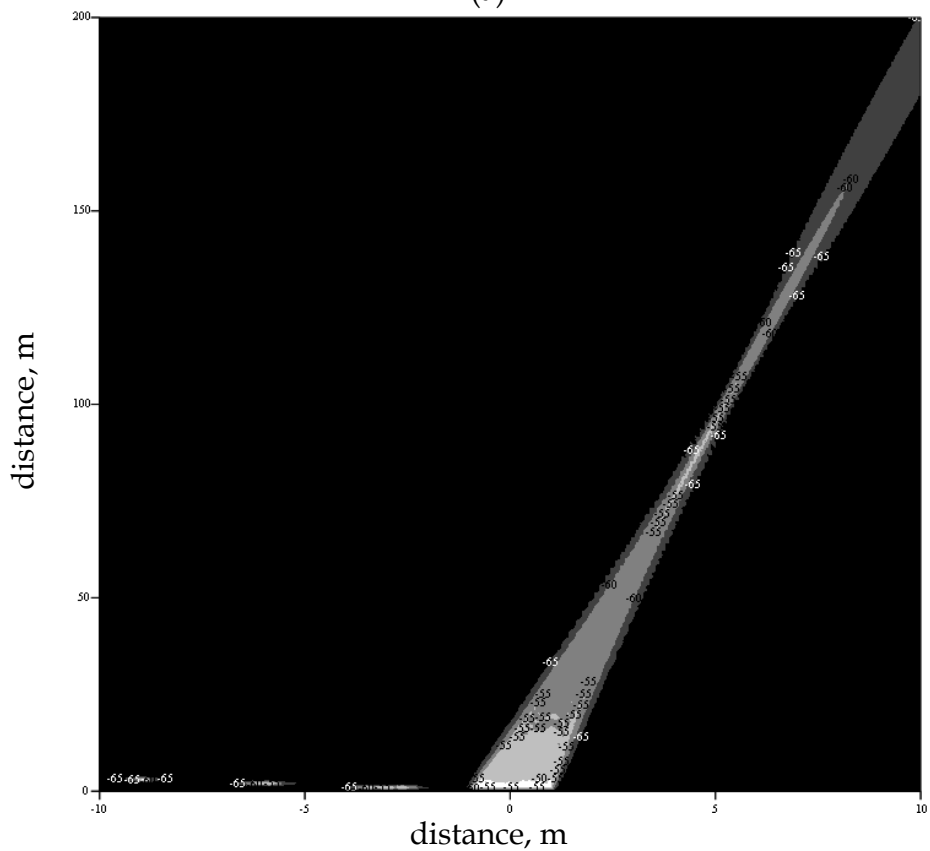

(b)

Fig. 14. Radiation patterns intersection in the azimuth plane of focused LAESR 


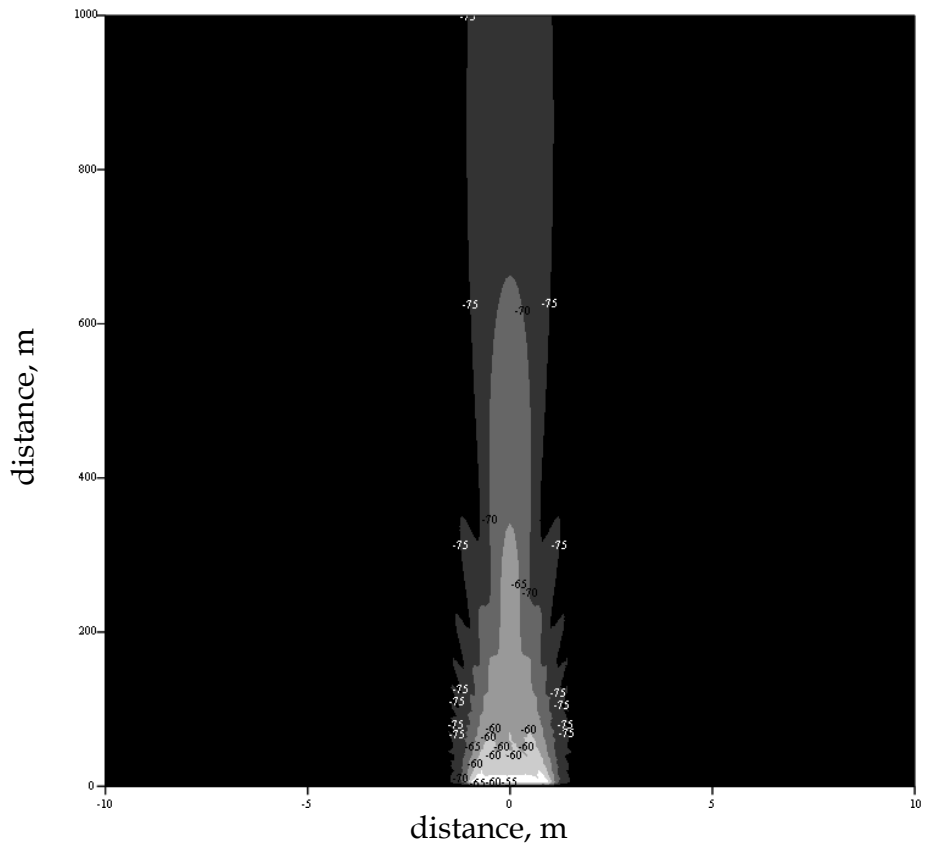

(a)

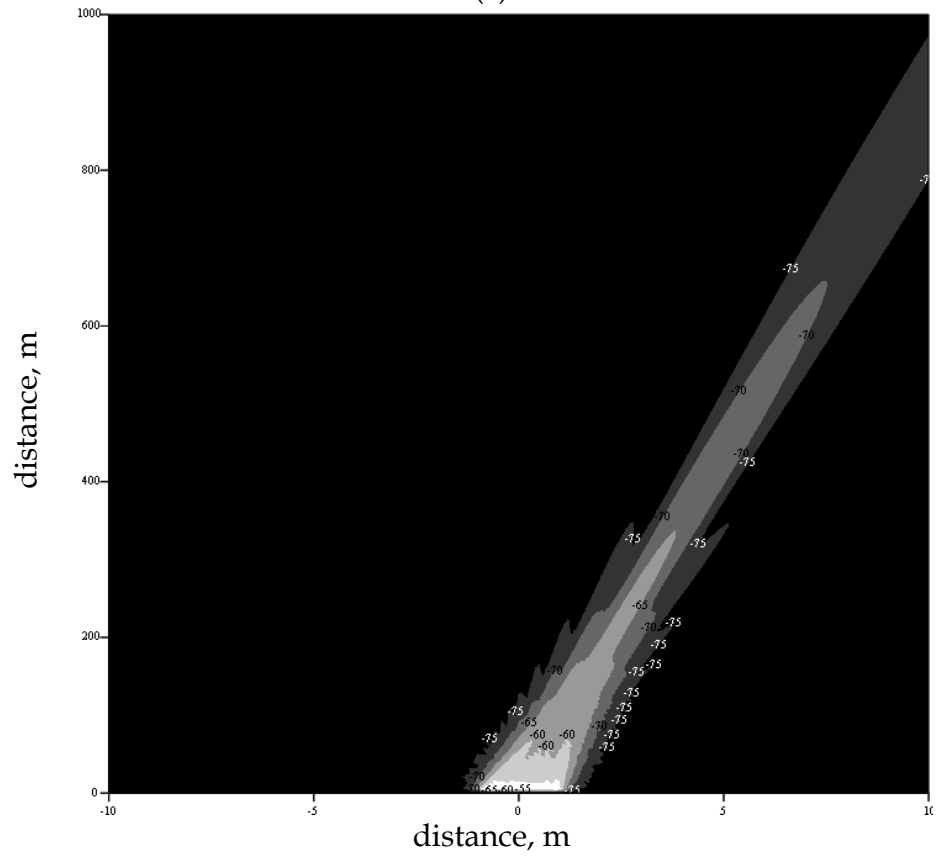

(b)

Fig. 15. Radiation patterns intersection in the azimuth plane of focused LAESR 


\section{Conclusion}

In this chapter, beamforming of antenna arrays focused in NFZ or IFZ is studied and the new principles of this process are revealed with a purpose to increase the 3-dimensional gain performance of antenna arrays at a wide range of angles and to improve the quality level of this technique for expanding applicability of the focused antenna.

The antenna arrays of linear and polygonal structure with different location and types of array elements have been considered in this paper, so all tasks of study of FAA radiation pattern synthesis have done in its azimuth plane or in polar coordinates.

Expressions of spatial shifts between elements and phase center of arrays where spatial shifts by distance and spatial shifts by angular coordinate are obtained as two separate parts of one equation without any approximation (Fresnel or Fraunhofer) are introduced. These equations had been obtained first time and they can be useful for FAA with separate phase steering by distance and by angular coordinate synthesis, it also helps to reduce the level of computational operations to calculate required phase distribution in array elements.

Radiation pattern synthesis and focusing properties of focused arrays are considered in this work, which are based on obtained geometrical models of arrays of different structures. Location of array phase center, different signal attenuations by propagation from array elements to observation point, different angles of radiation from array elements to observation point and array elements radiation patterns dependence on the distance must be taken to account for precise beamforming of arrays focused in NFZ or IFZ. The principles of radiation pattern synthesis of arrays focused in NFZ or IFZ and arrays focused in FFZ are equal. The properties of radiation pattern by distance and angular coordinate are equal too.

The main focusing properties of antenna array are based on principle of inversion of the Fresnel and the Fraunhofer diffraction fields which is the result of focusing process. The applicability of arrays is limited by hyperfocal distance and signal attenuation by propagation. The focal area or a certain segment of distance $R \in\left[0, R_{F}\right]$ where radiated power distribution is uniform can be the result of focusing process.

FAA directivity by distance as angular directivity in focused point to angular directivity in FFZ ratio is introduced in this chapter.

FAA directivity can be improved by increasing array elements spacing jointly with grating focal area supressing or by excitation of array elements by wideband signals or by using of modified APD in array elements and modified AFC of excited wideband signal which are introduced in this chapter. The spectrum of spatial frequencies concept was used for FAA directivity improvement.

Investigation results of possible structures of FAA which capable to three-dimensional spatial division multiplexing in all frequency ranges used by a modern radio-technique are presented in this work. These results have shown the given FAA applicability in a radio and short range telecommunication engineering.

The task of obtaining a exact expression for radiation pattern of antenna array without any approximation still remain unsolved due to lack of solution in the form of a simple or a special function. Beamforming of planar array using study results achieved in this chapter have not considered. Methods of FAA directivity improving are considered more qualitatively than quantitatively. Mutual coupling effect among the array elements and its real dimensions is not assumed. These facts reveal the possibility of further research. 


\section{References}

Buffi A.; Serra A.; Nepa P.; Manara G. \& Luise M. (2009). Near field focused microstrip arrays for gate access control systems, 2009 IEEE International Antennas and Propagation Symp. Dig., vol. 47, (June 2009), pp. 1704 - 1707

Chu T. S. (1971). A note on simulating Fraunhofer radiation patterns in the Fresnel region, Antennas and Propagation, IEEE Transactions on, vol. 19, (Sept. 1971), pp. 691 - 692

Fenn A. J. (2007). Adaptive Antennas and Phased Arrays for Radar and Communications. Artech House Publishers, ISBN: 978-1-59693-273-9, Boston, USA

Garrett J. E. \& Wiltse J. C. (1990). Antenna pattern characteristics of phase-correcting Fresnel zone plates, 1990 IEEE International Antennas and Propagation Symp. Dig., vol. 28, (May 1990), pp. 1906 - 1909

Graham W. J. (1983). Analysis and synthesis of axial field patterns of focused apertures, Antennas and Propagation, IEEE Transactions on, vol. 31, (July 1983), pp. 665 - 668

Hansen R. C. (1964). Microwave Scanning Antennas. vol. 1, Apertures., Ed. Academic Press, New York, USA

Hansen R. C. (1985). Focal region characteristics of focused array antennas, Antennas and Propagation, IEEE Transactions on, vol. 33, (December 1985), pp. 1328 - 1337

Hansen R. C. (2009). Phased Array Antennas, 2nd Ed., John Wiley \& Sons, ISBN 978-0-47040102-6, New Jersey, USA

Herben M. H. A. J. \& Hristov H. D. (1999). Some developments in Fresnel zone plate lens antennas, 1999 IEEE International Antennas and Propagation Symp. Dig., vol. 37, (June 1999), pp. $726-729$

Hristov H. D.; Feick R.; Grote W. \& Fernández P. (2004). Indoor signal focusing by means of Fresnel zone plate lens attached to building wall, Antennas and Propagation, IEEE Transactions on, vol. 52, (April 2004), pp. 933 - 940

Hussain M. G. M. \& Al-Zayed A.S. (2008). Aperture-sparsity analysis of ultrawideband twodimensional focused array, Antennas and Propagation, IEEE Transactions on, vol. 56, (July 2008), pp. 1908 - 1918

Hussain M. G. M. (2004). Characteristics of ultrawideband electro-magnetic missiles generated by focused two-dimensional array. Progress In Electromagnetics Research, PIER 49, pp.143-159

Ishimaru A.; Jaruwatanadilok S. \& Kuga Y. (2007). Imaging of a target through random media using a short-pulse focused beam, Antennas and Propagation, IEEE Transactions on, vol. 55, (June 2007), pp. 1622 - 1629

Karimkashi Sh. \& Kishik A. A. (2008). A New Fresnel Zone Antenna with beam Focused in the Fresnel Region. U.R.S.I. XXIX General Assembly 7-16 August 2008, Chicago, USA. Available from:http://ursi-test.intec.ugent.be/files/URSIGA08/papers/BP7p7.pdf

Karimkashi, Sh. \& Kishk, A.A. (2009). Focused Microstrip Array Antenna Using a DolphChebyshev Near-Field Design, Antennas and Propagation, IEEE Transactions on, vol.57, no.12, (December 2009), pp.3813-3820

Korostelev A. A. (1987). Space-time theory of radio system. Radio i Svyaz, Moscow, USSR (in Russian)

Kremer I. Ya., Kremer A. I. \& Petrov V. M. (1984). Space-time signal processing. Radio i Svyaz, Moscow, USSR (in Russian) 
Laybros S.; Combes P. F. \& Mametsa H. J. (2005). The "Very-near-field"region of equiphase radiating apertures, IEEE Antennas and Propagation Magazine, vol. 47, (August 2005), pp. $50-66$

Malyuskin O. \& Fusco V. (2009). Near field focusing properties of finite wire array under sinusoidal pulse excitation, 2009 IEEE International Antennas and Propagation Symp. Dig., vol. 47, (June 2009), pp. 3380 - 3383

Mazurenko, A.V. \& Yakornov, E.A. (2010). Analysis of perspective antenna systems with controlled 3-dimensional signal space division, Microwave and Telecommunication Technology (CriMiCo), 2010 20th International Crimean Conference, vol.2, pp.562-563, 13-17 Sept. 2010

Molotkov N. Ya., Lomakina O. V. \& Yegorov A. A. (2009). UHF optics and quasioptics. Publishing of TSTU, ISBN 978-5-8265-0880-0, Tambov, Russia. (in Russian)

Narasimhan M. S. \& Philips B. (1987a). Synthesis of near-field patterns of arrays, Antennas and Propagation, IEEE Transactions on, vol. 35, (February 1987), pp. 212 - 218

Narasimhan M. S. \& Philips B. (1987b). Synthesis of near-field patterns of a nonuniformly spaced array, Antennas and Propagation, IEEE Transactions on, vol. 35, (November 1987), pp. 1189 - 1198

Polk Ch. (1956). Optical fresnel-zone gain of a rectangular aperture, Antennas and Propagation, IRE Transactions on, vol. 4, (January 1956), pp. 65 - 69

Reid D. R. \& Smith G. S. (2009). A comparison of the focusing properties of a Fresnel zone plate with a doubly-hyperbolic lens for application in a free-space, focused-beam measurement system, Antennas and Propagation, IEEE Transactions on, vol. 57, (February 2009), pp. $499-507$

Rudolph S. M. \& Grbic A. (2008). Super-resolution focusing using volumetric, broadband NRI media, Antennas and Propagation, IEEE Transactions on, vol. 56, (September 2008), pp. 2963 - 2969

Wang G. ; Fang J. \& Dong X. (2007). Resolution of near-field microwave target detection and imaging by using flat LHM lens, Antennas and Propagation, IEEE Transactions on, vol. 55, (December 2007), pp. 3534 - 3541

Wu, T. T. (1985). Electromagnetic missiles, Journal of Applied Physics, vol. 57, pp. 2370-2372.

Xiao Shu, Schoenbach K.H. \& Baum C. E. (2008). Time-Domain Focusing Radar for Medical Imaging. U.R.S.I. XXIX General Assembly 7-16 August 2008, Chicago, USA. Available from:http:// ursi-test.intec.ugent.be/files/URSIGA08/papers/E02p4.pdf 


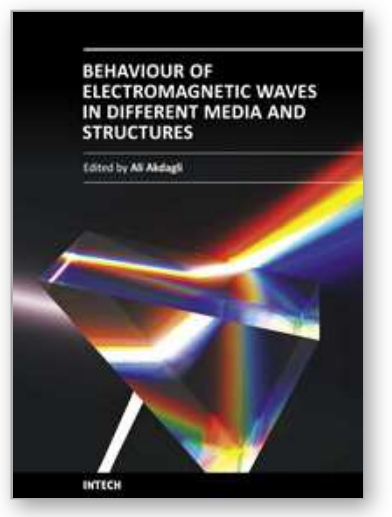

\section{Behaviour of Electromagnetic Waves in Different Media and Structures \\ Edited by Prof. Ali Akdagli}

ISBN 978-953-307-302-6

Hard cover, 440 pages

Publisher InTech

Published online 09, June, 2011

Published in print edition June, 2011

This comprehensive volume thoroughly covers wave propagation behaviors and computational techniques for electromagnetic waves in different complex media. The chapter authors describe powerful and sophisticated analytic and numerical methods to solve their specific electromagnetic problems for complex media and geometries as well. This book will be of interest to electromagnetics and microwave engineers, physicists and scientists.

\section{How to reference}

In order to correctly reference this scholarly work, feel free to copy and paste the following:

Oleksandr Mazurenko and Yevhenii Yakornov (2011). Focused Arrays Beamforming, Behaviour of Electromagnetic Waves in Different Media and Structures, Prof. Ali Akdagli (Ed.), ISBN: 978-953-307-302-6, InTech, Available from: http://www.intechopen.com/books/behavior-of-electromagnetic-waves-in-differentmedia-and-structures/focused-arrays-beamforming1

\section{INTECH}

open science | open minds

\author{
InTech Europe \\ University Campus STeP Ri \\ Slavka Krautzeka 83/A \\ 51000 Rijeka, Croatia \\ Phone: +385 (51) 770447 \\ Fax: +385 (51) 686166 \\ www.intechopen.com
}

\author{
InTech China \\ Unit 405, Office Block, Hotel Equatorial Shanghai \\ No.65, Yan An Road (West), Shanghai, 200040, China \\ 中国上海市延安西路65号上海国际贵都大饭店办公楼 405 单元 \\ Phone: +86-21-62489820 \\ Fax: $+86-21-62489821$
}


(C) 2011 The Author(s). Licensee IntechOpen. This chapter is distributed under the terms of the Creative Commons Attribution-NonCommercialShareAlike-3.0 License, which permits use, distribution and reproduction for non-commercial purposes, provided the original is properly cited and derivative works building on this content are distributed under the same license. 